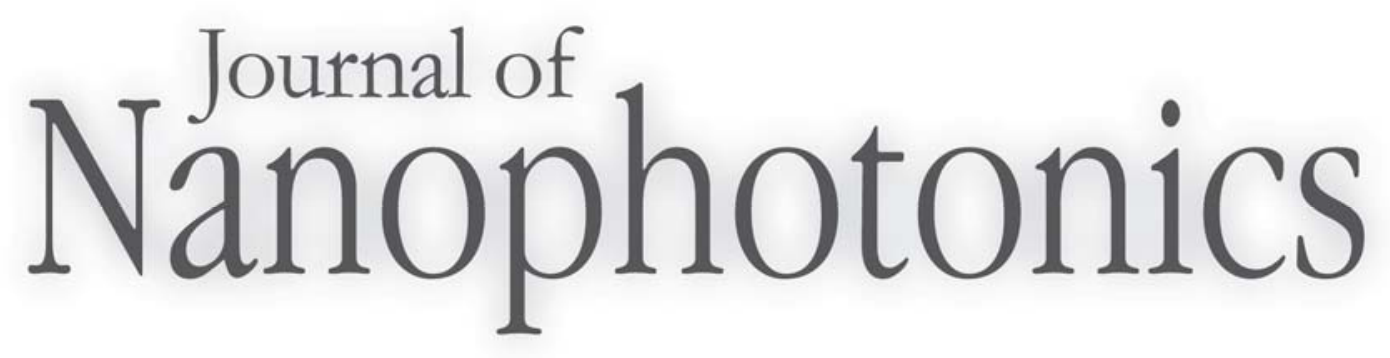

SPIEDigitalLibrary.org/jnp

\title{
Near-field intensity correlations on nanoscaled random silver/dielectric films
}

Julien Laverdant

Stéphanie Buil

Jean-Pierre Hermier

Xavier Quélin 


\title{
Near-field intensity correlations on nanoscaled random silver/dielectric films
}

\author{
Julien Laverdant, a Stéphanie Buil, ${ }^{\text {b Jean-Pierre Hermier, }}$ \\ and Xavier Quélin ${ }^{b}$ \\ ${ }^{a}$ Université Pierre et Marie Curie, CNRS UMR7588, Institut des Nanosciences de Paris, \\ Campus Boucicaut, 140 rue de Lourmel, 75015 Paris, France \\ jlaverda@club-internet.fr \\ ${ }^{\mathrm{b}}$ Université de Versailles Saint-Quentin, Groupe d'Etudes de la Matière Condensée (UMR \\ CNRS/UVSQ 8635), 45 avenue des Etats-Unis, 78035 Versailles cedex, France \\ buil@physique.uvsq.fr
}

\begin{abstract}
Near-field intensity distributions in inhomogeneous silver nanostructures were investigated using near-field optical microscopy. By varying the metallic concentration and the excitation wavelength, different plasmon scattering regimes were addressed and identified using statistical analysis tools such as the probability density function and intensity autocorrelation function. The intensity correlation length was found to indicate an intermediate regime where both surface scattering and plasmon localization coexist on the same scale.
\end{abstract}

Keywords: Near-field optics, random media, plasmonics.

\section{INTRODUCTION}

The optical properties of random nanoscaled metal-dielectric thin films have attracted considerable interests these last years [1]. In these inhomogeneous structures, it is well-known that the incident light can excite Surface Plasmon Resonances (SPR). For a peculiar metallic concentration regime near $p_{c}$, corresponding to the threshold between insulator and conductor samples, these SPR can lead to strong energy localization on subwavelength areas, usually called hot spots [2]. This localization has been identified to an Anderson-type localization of the SPR. On these hot spots, the electromagnetic field can be locally enhanced by several orders of magnitude compared to the incident field [1]. Scanning Near-Field Optical Microscopy (SNOM) is the experimental key to give access to the localized fields: it allows to detect non-propagating fields with a subwavelength resolution [2]. Using near-field optical images of the intensity distribution on random metal-dielectric thin films, statistical analysis on experimental data have been recently performed to underline some specific behaviors. The power-law dependence of the Probability Density Function (PDF) for the intensity enhancement has been identified as a fingerprint of the scale invariance of such systems [3]. The spatial intensity Auto-Correlation Function (ACF), a second-order correlation function representative of the coherence of the intensity statistical distribution [4,5], has been deduced from experimental and/or numerical data to determine the correlation length $[6,7,8]$ and the influence of the incident polarization $[9,10]$.

In this letter, we present an experimental study of the PDF and of the near-field intensity correlations on nanoscaled metal-dielectric films. The metallic concentration $p$ on a dielectric substrate and the incident light wavelength $\lambda$ are varied to address different scattering regimes. Deduced from the intensity data collected by the use of an aperture SNOM, we examine the PDF for different scattering regimes. Then the intensity ACF is calculated from experimental data to deduce the correlation length $R_{c}$. The evolution of the correlation length $R_{c}(p)$ is then studied and indicates an intermediate regime where both surface scattering and plasmon localization coexist on the same scale. 


\section{SAMPLE PREPARATION AND CHARACTERIZATION}

The metal-dielectric samples are obtained by RF-sputtering at room temperature. The silver nanoparticles, randomly deposited on the glass substrate, display a broad distribution of sizes and shapes as the surface coverage $p$ is increasing from low concentration to the vicinity of $p_{c}$. Far-field optical measurements have been performed using a Perkin-Elmer Lambda 950 spectrophotometer. The absorption spectra are displayed in Fig. 1 to show the different plasmon regimes for each concentration.

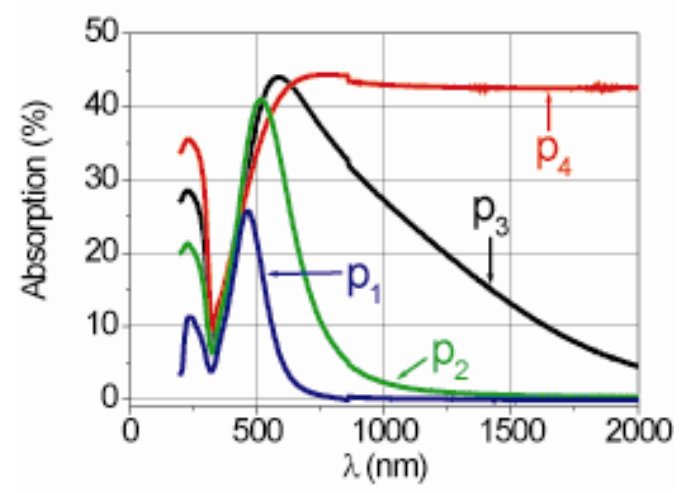

Fig. 1. Far-field absorption spectra of silver nanostructures for 4 different metallic concentrations $\left(p_{1} \approx 0.35, p_{2} \approx 0.45, p_{3} \approx 0.5\right.$ and $p_{4} \approx 0.6$ ).

At low metallic concentration, the metal nanoparticles are dispersed and the absorption spectrum exhibits a rather narrow peak (around $480 \mathrm{~nm}$ ) which is attributed to single SPR (Fig. 1, $p_{l}$ ). The nanoparticles (diameter of $20 \mathrm{~nm}$ ) are weakly interacting with each others which only results in a red-shift of the plasmon resonance. For intermediate values of metallic concentrations, the resonance becomes broader and is red-shifted $\left(p_{2}, p_{3}\right)$. This behavior is due to the appearance of interconnected metal clusters with increasing metallic concentration $p$, which broadens the size and shape distributions of resonant nanoparticles. Near the percolation threshold $p_{c}$, a large flat broad band $\left(p_{4}\right)$ of plasmonics absorption is observed and is usually attributed to the fractal film structure: clusters of all sizes and shapes exist and support a wide range of resonance frequencies (see Ref. 11 and references cited therein). In accordance with the spectral absorption curves, the metallic concentrations have been deduced: for samples $1,2,3$ and 4 , the concentrations are $p_{1} \approx 0.35, p_{2} \approx 0.45, p_{3} \approx 0.5$ and $p_{4} \approx 0.6$ respectively. The percolation threshold is estimated to be $p_{c} \approx 0.65$.

\section{EXPERIMENTAL SETUP AND RESULTS}

The experimental data of the near-field intensities on nanostructured silver samples have been obtained using a commercial SNOM (Veeco, Aurora 2). This is an aperture-type SNOM operating with Al-coated optical fibers with an aperture of $50 \mathrm{~nm}$. The tip fiber is regulated at constant distance $(\sim 10 \mathrm{~nm})$ from the surface sample through shear-force feedback. The experiments were performed one after the other on the same sample by changing the excitation wavelength at each time. All the results have been obtained in transmission/collection mode and under normal incidence [12].

When the sample is illuminated, on one hand the light is scattered by the surface roughness and on the other hand, the light is coupled to SPR. The SPR can be localized and scattered once more by the surface roughness $[3,12]$. Therefore, near-field intensities result in a combination of the scattering due to the structural inhomogeneities and called surface 
scattering, and plasmon scattering. For the illumination, three $\mathrm{Ar} / \mathrm{Kr}$ laser lines were used: $\lambda_{l}$ $=514 \mathrm{~nm}, \lambda_{2}=568 \mathrm{~nm}$, and $\lambda_{3}=647 \mathrm{~nm}$.

$\Gamma$

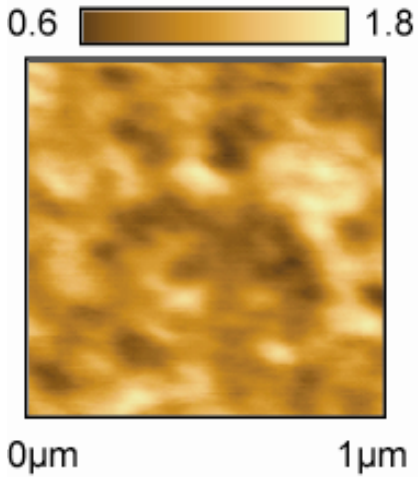

(a)

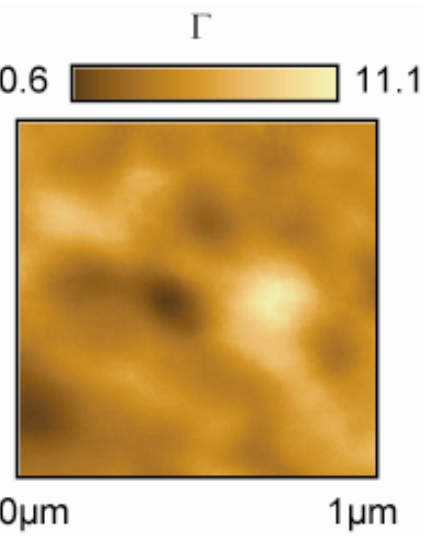

(b)

Fig. 2. Near-field optical images obtained in transmission/collection mode under normal incidence for: (a) sample with $p_{l} \approx 0.35$ at $\lambda_{I}=514 \mathrm{~nm}$; (b) sample with $p_{4} \approx 0.6$ at $\lambda_{3}=647 \mathrm{~nm}$.

Typical near-field images are presented in Fig. 2. The obtained intensity [Fig. 2(a)] measurements for $p_{I}$ at $\lambda_{I}$ (weak plasmon scattering regime) exhibit a rather narrow variation (from $\Gamma=0.6$ to 1.8 ). In Fig. 2(b), the intensity distribution for $p_{4}$ at $\lambda_{3}$ (strong plasmon scattering regime associated to plasmon localization) appears with some high intensity values (up to 11) in wide regions of weak intensity variations. One can notice the very satisfying quality of these images and we estimated the tip resolution to be far less than $100 \mathrm{~nm}$. Deduced from these $300 \times 300$ near-field intensities data points, we have first deduced the PDF of the intensity enhancement $\Gamma$.

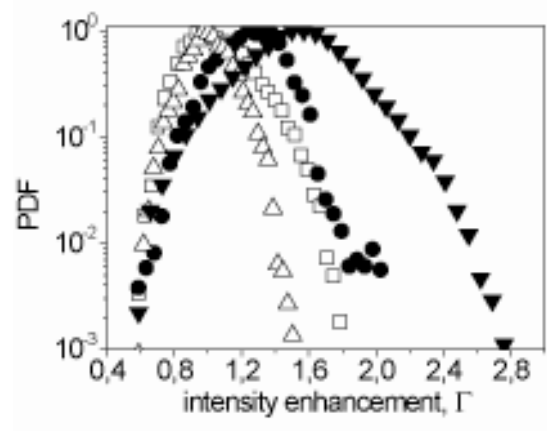

(a)

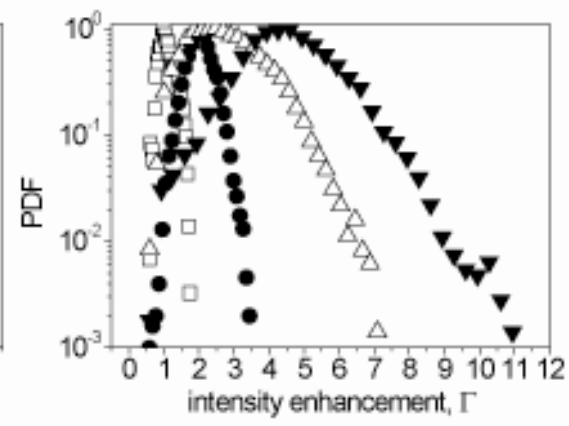

(b)

Fig. 3. Near-field Probability Density Functions (PDF) of the local field intensity enhancement $\Gamma$ on inhomogeneous silver films at $p_{1} \approx 0.35(\square), p_{2} \approx 0.45(\bullet)$, $p_{3} \approx 0.5(\Delta)$ and $p_{4} \approx 0.6(\boldsymbol{\nabla})$ for $\lambda_{1}=514 \mathrm{~nm}$ (a) and $\lambda_{3}=647 \mathrm{~nm}$ (b) respectively.

In Fig. 3, two PDFs are presented for all the four samples and for two excitation wavelengths $\lambda_{1}$ [Fig. 3(a)] and $\lambda_{3}$ [Fig. 3(b)]. In Fig. 3(a), one can notice that, for $p \leq p_{3}$, the PDFs are rather symmetric and centered around $\Gamma=1$ while, for $p=p_{4}$, the PDF is extended to larger (but less than 3 ) values of $\Gamma$. According to the spectra presented in Fig. 1 and to Ref. 6 , one can consider that, at the excitation wavelength $\lambda_{1}$, all samples are in a surface plasmon 
resonance regime: for $p_{1}$ to $p_{3}$ concentrations, this corresponds to weak single-SPR regime and for the concentration $p_{4}$, this regime is in-between weak plasmon scattering and weak plasmon localization. In Fig. 3(b) at $\lambda_{3}$, one can notice that, if for the $p_{1}$ concentration, the PDF is still narrow and symmetric, centered at the value $\Gamma=1$, for $p_{2}$ to $p_{4}$ concentrations, the PDFs become more and more asymmetric (leading to log-normal functions) and are broadened to larger and larger values of $\Gamma$, up to $\Gamma=11$. This can be interpreted as a crossover between weak and strong plasmon scattering. Large values of electromagnetic enhancements in the PDFs correspond to the presence of hot spots in the optical images and are one of the feature of strong scattering, leading to strong localization. The different scattering regimes are now identified, depending on the metallic concentration and the excitation wavelength considered.

\section{STATISTICAL ANALYSIS AND DISCUSSION}

One relevant tool to study fluctuations of the in-plane intensity distributions is the intensity correlator $\mathrm{C}(\mathrm{R})$, also called the intensity ACF which is defined as:

$$
C(R)=\langle\delta I(r) \cdot \delta I(r+R)>
$$

where the angular brackets denote statistical averaging on the in-plane position $r$ and the normalized intensity variations $\delta \mathrm{I}(\mathrm{r})$ are calculated with:

$$
\delta I(r)=[I(r)-<I(r)>] /<I(r)>.
$$

Again from the $300 \times 300$ near-field intensity data points, we have deduced the intensity ACF and we present in Fig. 4 the obtained function maps for $\left(p_{1}, \lambda_{l}\right)$ [Fig. 4(a) $]$ and for $\left(p_{4}\right.$, $\left.\lambda_{3}\right)$ [Fig. 4(b)].

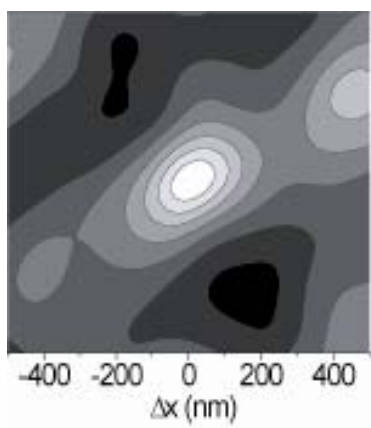

(a)

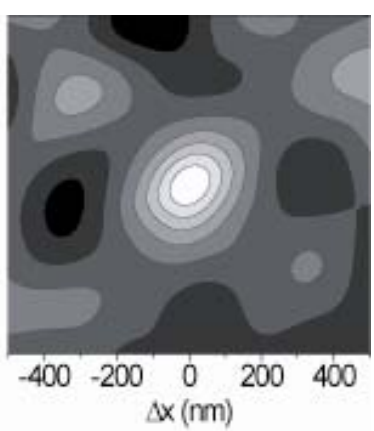

(b)

Fig. 4. 2D spatial intensity autocorrelation function maps for two different metallic coverages $p$ and two excitation wavelengths: (a) $p_{1} \approx 0.35$ and $\lambda_{1}=514 \mathrm{~nm}$; (b) $p_{4} \approx 0.6$ and $\lambda_{3}=647 \mathrm{~nm}$.

From these two representative graphs, it is clear that the central peak at $r=0$, representing the spatial energy distribution, is nearly isotropic and according to its width, the intensity is correlated on sub-wavelength scales. Then, in order to compare the correlation width at different surface coverage $p$, we define a correlation radius $R_{c}$ as the Half-Width at Half Maximum (HWHM) of the central peak of the intensity ACF for all 12 pairs $(p, \lambda)$. The obtained set of results $R_{c}(p)$ is presented in Fig. 5. 


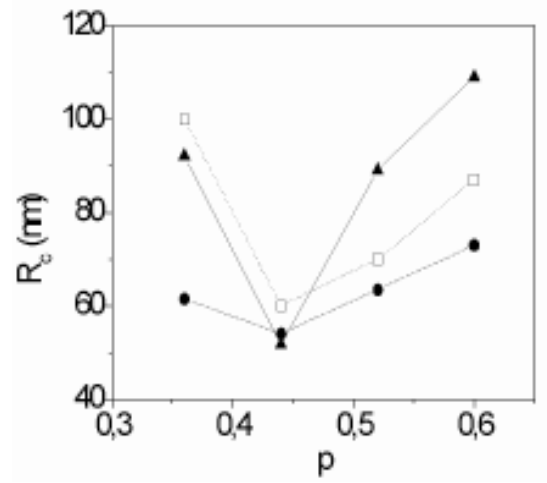

Fig. 5. Correlation radius $R_{c}$ of near-field intensity distributions as function of the metallic concentration $p$ for 3 different excitation wavelengths: $\lambda_{I}=514 \mathrm{~nm}(\bullet)$, $\lambda_{2}=568 \mathrm{~nm}(\square), \lambda_{3}=647 \mathrm{~nm}(\boldsymbol{\Delta})$.

For the lowest presented metallic concentration $p_{1}$, the values of the correlation radius are ranging from $61.5 \mathrm{~nm}$ to $100 \mathrm{~nm}$. We have previously deduced from the PDFs that, for the three excitation wavelengths, this sample was in single-SPR leading to weak plasmon scattering, together with surface scattering. As mentioned in Ref. 13 in the case of pure surface scattering on dielectric inhomogeneous medium, the correlation length is increasing with the wavelength. This behavior according to the excitation wavelength is quite well verified for $p_{l}$ and is also in agreement with the results presented in Fig. 1. The sample at $p_{1}$ is less in the single-SPR regime when $\lambda$ is increasing which means that, in our case, the surface scattering becomes predominant with increasing wavelength for this metallic concentration.

For the highest surface coverage $p_{4}$, the measured correlation radii are ranging from $73 \mathrm{~nm}$ to $109 \mathrm{~nm}$. The more the sample is in the SPR regime with strong scattering and strong localization associated, the longer is the correlation length which is a typical behavior of purely surface scattering. In other words, this result is in good agreement with what can be deduced from the PDF [Fig. 3(b)]: high intensity enhancement factors are rare and so, the hot spots spatial extension has only a weak influence on the intensity correlation length.

The unintuitive and surprising result appears in the intermediate metallic concentration regime. For the surface coverage $p_{2}$, the correlation radius values are concentrated, ranging from $52 \mathrm{~nm}$ to $60 \mathrm{~nm}$. For the set of 3 wavelengths used, these correlation lengths are the lowest values deduced from our experimental data. At this metallic surface coverage, the PDF appears in an intermediate behavior between $p_{l}$ and higher concentrations. This means that the PDF becomes broader (higher enhancement factors appear) with increasing wavelength, but remains nearly symmetric unlike higher concentrations. We assume that, for this intermediate concentration, the SPR scattering, which is not leading to strong localization, and the surface scattering are both responsible of the near-field intensity distribution.

\section{CONCLUSION}

In summary, different scattering regimes on random silver films have been addressed. Using an aperture SNOM and thanks to the very satisfying resolution of the tapered fiber, the nearfield intensity distribution has been measured. By the use of statistical analysis (PDF and intensity ACF), different scattering regimes have been identified. At low metallic concentration, single SPRs are involved and the near-field intensity distribution is dominated by surface scattering. Near the percolation threshold concentration, strong intensity localization appears but the near-field energy distribution is dominated again by the surface scattering. Between these two regimes, a critical metallic concentration has been identified. 
At this particular value the interaction between surface scattering and SPR-scattering leads to a minimum for the intensity correlation length.

\section{Acknowledgments}

This work is supported by the Centre National de la Recherche Scientifique, the University of Versailles-St-Quentin-en-Yvelines and the Region Ile-de-France (SESAME E.1677).

\section{References}

[1] V. A. Markel and T. F. Georges, Optics of Nanostructured Materials, Wiley Interscience, New York (2001).

[2] S. Grésillon, L. Aigouy, A. C. Boccara, J-C. Rivoal, X. Quélin, C. Desmarest, P. Gadenne, V. A. Shubin, A. K. Sarychaev, and V. M. Shalaev, "Experimental observation of localized optical excitations in random metal-dielectric films," Phys. Rev. Lett. 82, 4520-4523 (1999) [doi: 10.1103/PhysRevLett.82.4520].

[3] S. I. Bozhevolnyi and V. Coello, "Statistics of local field intensity enhancements at nanostructured surfaces investigated with a near-field optical microscope," Phys. Rev. B 64, 115414 (2001) [doi: 10.1103/PhysRevB.64.115414].

[4] J. W. Goodman, Statistical Optics, Wiley, New York (2000).

[5] E. Akkermans and G. Montambaux, Mesoscopic Physics of Electrons and Structures, Cambridge University Press, Cambridge (2007).

[6] K. Seal, A. K. Sarychev, H. Noh, D. A. Genov, A. Yamilov, V. M. Shalaev, Z. C. Ying, and H. Cao, "Near-field intensity correlations in semicontinuous metaldielectric films," Phys. Rev. Lett. 94, 226101 (2005) [doi: 10.1103/PhysRevLett.94.226101].

[7] A. Apostol and A. Dogariu, "Spatial correlations in the near field of random media," Phys. Rev. Lett. 91, 093901 (2003) [doi: 10.1103/PhysRevLett.91.093901].

[8] A. R. McGurn and A. Maradudin, "Intensity correlation function for light elastically scattered from a randomly rough metallic grating," Phys. Rev. B 39, 13160 (1989) [doi: 10.1103/PhysRevB.39.13160].

[9] C. Liu and S. H. Park, "Anisotropy of near-field speckle patterns," Opt. Lett. 30, 1602-1604 (2005) [doi: 10.1364/OL.30.001602].

[10] J. Laverdant, S. Buil, B. Berini, and X. Quélin, "Polarization dependent near-field speckle of random gold films," Phys. Rev. B 77, 165406 (2008) [doi: 10.1103/PhysRevB.77.165406].

[11] V. M. Shalaev, Ed., Optical Properties of Nanostructured Random Media, Springer, New York (2002).

[12] S. Buil, J. Aubineau, J. Laverdant, and X. Quélin, "Local field intensity enhancements on gold semicontinuous films investigated with an aperture nearfield optical microscope in collection mode," J. Appl. Phys. 100, 063530 (2006) [doi: 10.1063/1.2335664].

[13] V. Emiliani, F. Intonti, M. Cazayous, D. S. Wiersma, and M. Colocci, "Near-field short range correlation in optical waves transmitted through random media, Phys. Rev. Lett. 90, 250801 (2003) [doi: 10.1103/PhysRevLett.90.250801]. 